\title{
Oblique Reflection of Electrons from a Potential Barrier in Heterostructures
}

\author{
P. PFEFFER* AND W. ZAWADZKI \\ Institute of Physics, Polish Academy of Sciences, al. Lotników 32/46, 02-668 Warsaw, Poland
}

(Received August 22, 2014)

\begin{abstract}
Reflection of electrons from a potential barrier in heterostructures is described. An electric field of the barrier causes spin splitting of electron energies via the spin-orbit interaction and its form is calculated in the three-level $\boldsymbol{k} \cdot \boldsymbol{p}$ model for a nontrivial case of unbound electrons. It is shown that if the potential barrier is the only source of the spin-orbit interaction, the spin-flip electron reflections are not possible. However, there appear two unexpected possibilities related to the spin-orbit interaction: (a) non-attenuated electron propagation in the barrier whose height exceeds the energies of incoming electrons, (b) total reflection of electrons whose energies exceed barrier's height. It is indicated that the system can serve as a source of spin-polarized electron beams.
\end{abstract}

DOI: $10.12693 /$ APhysPolA.126.820

PACS: 71.70.Ej, 73.22.Dj, 73.40.-c, 75.76.+j

\section{Introduction}

It is well known that the spin splitting (SS) of energies in various quantum systems is intimately related to their symmetry. According to the Kramers theorem, the energy of an electron in a periodic system satisfies the equality: $E_{\boldsymbol{k} \uparrow}=E_{-\boldsymbol{k} \downarrow}$, where $\boldsymbol{k}$ is the wave vector and the arrows $\uparrow$ and $\downarrow$ signify spin-up and spin-down projections, respectively. If, in addition, a system is characterized by the inversion symmetry, the two spin states are degenerate for any $\boldsymbol{k}$ value: $E_{\boldsymbol{k} \uparrow}=E_{\boldsymbol{k} \downarrow}$. In general, however, for a given $\boldsymbol{k}$ direction and value SS may occur even without an external magnetic field. It was shown by Dresselhaus [1] that in bulk semiconductors of the zinc blende structure, which have no inversion symmetry, the conduction bands of III-V compounds are characterized by an anisotropic spin splitting proportional to $k^{3}$ for small $k$ values. This splitting was extensively investigated in bulk semiconductors. With the advancement of semiconductor quantum structures a new type of inversion asymmetry became possible, namely the structure inversion asymmetry (SIA). The SIA mechanism also leads to SS of electron energies. The SS related to SIA, often called "the Rashba splitting", is of interest due to possible applications, as it can be influenced by an external electric bias. This interest is a part of the wider movement aimed to use properties of electron spin for technical use.

The subject of SS related to SIA has a controversial history, as reviewed by the present authors [2]. In a widely quoted paper, Bychkov and Rashba [3] wrote down the following Hamiltonian for SS in heterostructures, in which the growth direction is parallel to a high symmetry axis:

$$
\widehat{H}_{\mathrm{SIA}}=\alpha(\boldsymbol{\sigma} \times \boldsymbol{k}) \cdot \boldsymbol{\nu} .
$$

\footnotetext{
*corresponding author; e-mail: pfeff@ifpan.edu.pl
}

Here $\boldsymbol{\sigma}$ are the Pauli matrices, $\boldsymbol{k}$ is the $2 \mathrm{D}$ electron wave vector transverse to the growth direction, $\boldsymbol{\nu}$ is the unit vector in the growth direction, and $\alpha$ is a coefficient. In their paper, Bychkov and Rashba did not mention the inversion asymmetry, but it is recognized by now that the coefficient $\alpha$ has a nonzero value only if the system is characterized by a structure inversion asymmetry along the growth direction. The Hamiltonian given by Eq. (1) resembles that of the spin-orbit interaction (SOI), but one should not confuse it with the SOI in which an electric field $\mathcal{E}$ appears instead of $\boldsymbol{\nu}$. As follows from the description given in [2], a consensus on the theoretical treatment of the problem of SS due to SIA has been reached, see also [4-6]. Namely, it has been demonstrated that the SS of conduction energies in asymmetric quantum wells (QWs) is mostly related to asymmetric offsets of the conduction and valence bands, both due to different energy gaps and spin-orbit energies. In particular, the SS of a conduction band is not proportional to the electric field in this band. If this were the case, the SS would almost vanish because the average electric field in a bound state is zero if one neglects a difference of the effective masses in the well and barriers [7].

It was recognized a few years ago that the SOI can be used to manipulate electron spins in semiconductor heterostructures. In particular, it was proposed to fabricate spin filters by either driving electrons through inhomogeneous heterostructures with different strengths of the SOI $[8,9]$ or by reflecting $2 \mathrm{D}$ electrons from a lateral potential barrier in an asymmetric quantum well [10-15]. It was exprimentally demonstrated by Chen et al. [10, 12, 14] that, in the presence of the SOI, an opaque reflection from a potential barrier separates electrons undergoing spinflip reflections from those experiencing spin-conserving reflections. In their system, the electron spin splitting was caused by SIA of asymmetric QWs and the interaction with the barrier.

In our paper we consider reflection of unbound 2D electrons from a potential barrier in heterostructures with- 
out the "help" of SIA due to asymmetric quantum well. Thus, we consider the simple system in which the only source of electron spin-splitting is its interaction with the barrier. This can be realized either by using a symmetric $\mathrm{QW}$ or by performing experiments without $2 \mathrm{D}$ heterostructures. We actually calculate the spin splitting of unbound electron energies due to the interaction with the barrier, which was not attempted in previous works. It is shown that, if the barrier is the only source of the SOI, the topology of the system does not allow for spinflip reflection processes. However, we point out quite unexpected possibilities of non-attenuated electron propagation in the barrier whose height exceeds the energies of incoming electrons and the total reflection of electrons whose energies exceed barrier's height. It is argued that even this simple system can be used as a source of spinpolarized electron beams.

The paper is organized as follows. In Sect. 2 we consider the spin splitting of electron energies due to the interaction with the barrier. Section 3 contains analysis of opaque reflections: their kinematics and amplitudes. Section 4 gives numerical estimations of the results. In Sect. 5 we discuss our approach as well as those of other authors. The paper is concluded by a Summary.

\section{Spin splitting due to potential barrier}

We first calculate the spin splitting of electron energies caused by the spin-orbit interaction between the incoming electron and the potential barrier. The interaction is of the general form given by Eq. (1) but the problem in our case is not trivial since the energy spectra of unperturbed and perturbed energies are continuous. We consider a finite potential barrier at $z=0$. The starting point for the theory is the multiband $\boldsymbol{k} \cdot \boldsymbol{p}$ formulation including the potential $V(\boldsymbol{r})$ on the diagonal

$$
\begin{aligned}
& \sum_{l}\left[\left(-E+V+\varepsilon_{l 0}+\frac{p^{2}}{2 m_{0}}\right) \delta_{l^{\prime} l}+\frac{1}{m_{0}} \boldsymbol{p}_{l^{\prime} l} \cdot \boldsymbol{p}\right] \\
& \quad \times f_{l}(\boldsymbol{r})=0,
\end{aligned}
$$

where $l^{\prime}=1,2, \ldots, E$ is the electron energy, $\varepsilon_{l 0}$ are the band edge energies, $m_{0}$ is the free electron mass, $f_{l}(\boldsymbol{r})$ are the envelope functions, and $\boldsymbol{p}_{l^{\prime} l}$ are the interband matrix elements of momentum taken between the Luttinger-Kohn periodic amplitudes $u_{l}(\boldsymbol{r})$. The potential $V(\boldsymbol{r})$ and the envelope functions $f_{l}(\boldsymbol{r})$ are assumed to be slowly varying within the unit cell (which is not always a realistic assumption). If the potential varies only in one dimension: $V(\boldsymbol{r})=V(z)$, one may separate the variables by looking for solutions in the form $f_{l}(\boldsymbol{r})=\exp \left(\mathrm{i} k_{x} x+\mathrm{i} k_{y} y\right) \chi_{l}(z)$. This gives $p_{x} \rightarrow \hbar k_{x}$, $p_{y} \rightarrow \hbar k_{y}$, and the only nontrivial variable is $z$. Within the three-level model (3LM) one deals with 8 bands arising from $\Gamma_{6}^{\mathrm{c}}, \Gamma_{8}^{\mathrm{v}}$ (double degenerate) and $\Gamma_{7}^{\mathrm{v}}$ levels. Thus the sum over $l$ in Eq. (2) runs from 1 to 8 and $l^{\prime}=1, \ldots, 8$, so that one deals with 8 coupled differential equations for the functions $\chi_{l}(z)$. The parameters in set (2) are the band edge energies and the momentum matrix elements. Within 3LM there is one nonvanishing matrix element $P_{0}$ between $\Gamma_{6}^{\mathrm{c}}$ and $\Gamma_{8}^{\mathrm{v}}, \Gamma_{7}^{\mathrm{v}}$ symmetry functions and one spin-orbit energy $\Delta$ splitting the $\Gamma_{8}^{\mathrm{v}}$ and $\Gamma_{7}^{\mathrm{v}}$ functions. Using minor approximations one reduces set (2) by substitution to two equations for the effective spin-up and spin-down conduction states, see Ref. [2]. The resulting eigenvalue problem for the growth direction $z$ parallel to [001] crystal axis is

$$
\left(\begin{array}{cc}
\widehat{A}-E & \widehat{K}_{\text {SIA }} \\
\widehat{K}_{\text {SIA }}^{\dagger} & \widehat{A}-E
\end{array}\right)\left(\begin{array}{l}
\chi_{1}(z) \\
\chi_{2}(z)
\end{array}\right)=0,
$$

where

$$
\widehat{A}=-\frac{\hbar^{2}}{2} \frac{\partial}{\partial z} \frac{1}{m^{*}} \frac{\partial}{\partial z}+\frac{\hbar^{2} k_{\perp}^{2}}{2 m^{*}}+V(z),
$$

in which $k_{\perp}^{2}=k_{x}^{2}+k_{y}^{2}$ and $m^{*}(E, z)$ is the effective mass for the conduction electrons

$$
\frac{m_{0}}{m^{*}(E, z)}=1+C-\frac{E_{P}}{3}\left(\frac{2}{\tilde{\varepsilon}_{i}}+\frac{1}{\tilde{f}_{i}}\right),
$$

where we explicitly indicate by the subscripts that $\tilde{\varepsilon}_{i}$ and $\tilde{f}_{i}$ are different in various parts of the system. Further

$$
\widehat{K}_{\mathrm{SIA}}=\frac{-\mathrm{i} k_{-}}{\sqrt{2}} \frac{\partial \eta(z)}{\partial z},
$$

where

$$
\eta(z)=\frac{2 P_{0}^{2}}{3}\left(\frac{1}{\tilde{\varepsilon}_{i}}-\frac{1}{\tilde{f}_{i}}\right),
$$

in which $k_{-}=\left(k_{x}-\mathrm{i} k_{y}\right) / \sqrt{2}$, while $\tilde{\varepsilon}_{i}(z)=\varepsilon_{i}(z)+$ $V(z)-E$ and $\tilde{f}_{i}(z)=\varepsilon_{i}(z)+\Delta_{i}(z)+V(z)-E$. Here $\varepsilon_{i}(z)$ are the energy gaps, $\Delta_{i}(z)$ are the spin-orbit energies, $E_{P}=P_{0}^{2} 2 m_{0} / \hbar^{2}$ and $C$ represents far-band contributions to the effective mass. The functions $\tilde{\varepsilon}_{i}(z)$ and $\tilde{f}_{i}(z)$ depend on $z$ not only via $V(z)$, but also due to the jumps of $\varepsilon_{i}$ and $\Delta_{i}$ at the interfaces. We consider the case of steep barrier: $V(z)=0, \varepsilon_{i}(z)=\varepsilon_{A}, \Delta_{i}(z)=\Delta_{A}$ and $\eta(z)=\eta_{A}$ for $z \leq 0$, while $V(z)=V_{b}, \varepsilon_{i}(z)=\varepsilon_{B}$, $\Delta_{i}(z)=\Delta_{B}$ and $\eta(z)=\eta_{B}$ for $z>0$, see Fig. 1 . We denote $m^{*}(E, z)=m_{A}^{*}$ on the left of the barrier and $m^{*}(E, z)=m_{B}^{*}$ on the right. Considering an electron coming to the barrier from an oblique direction one can choose, without loss of generality, the coordinate system in such a way that $k_{y}=0$, while $k_{x} \neq 0$ and $k_{z} \neq 0$.

It is seen that for $K_{\mathrm{SIA}}=0$ the two spin states have the same energy. We will solve set (3) using the well known approximate procedure for the perturbation of two degenerate states by calculating the matrix element of the nondiagonal term $K_{\text {SIA }}^{\uparrow \downarrow}$ between the solutions of diagonal terms. Explicitly

$$
E_{1,2}=\widehat{A}^{\uparrow \uparrow} \pm\left|\widehat{K}_{\text {SIA }}^{\uparrow \downarrow}\right| \text {. }
$$

The matrix element is taken between two orthogonal spin states $\chi_{\uparrow}(z)=(1,0)^{\mathrm{T}} \chi(z)$ and $\chi_{\downarrow}(z)=(0,1)^{\mathrm{T}} \chi(z)$, where $\chi(z)$ is a solution of the Schrodinger equation $\widehat{A} \chi(z)=E \chi(z)$, see Eq. $(3)$, and $(1,0)^{T}$ and $(0,1)^{\mathrm{T}}$ are the spin-up and spin-down eigenvectors of the Pauli spin operator $\widehat{\sigma}_{z}$, respectively. The unperturbed and perturbed electron spectrum is continuous and the wave functions are plane waves. This means that the wave functions along the $z$ direction must be normalized to 
the delta function $\delta\left(k_{z}-k_{z}^{\prime}\right)$. In consequence, the matrix elements $A^{\uparrow \uparrow}$ and $K_{\text {SIA }}^{\uparrow \downarrow}$ have the dimension of [energy $\times$ length]. In order to obtain the correct dimension of [energy] one has to integrate Eq. (8) over $k_{z}^{\prime}$. We first consider in more detail the unperturbed wave functions. There is, for each spin, an incoming and a reflected plane wave for $z \leq 0$ and a decaying wave for $z>0$. The incoming wave vector is $k_{z}>0$, the reflected one is $-k_{z}$ and the decaying wave is described by the imaginary wave vector $q_{z}$. The value of $k_{x}$ does not change since there is no force acting along the $x$ direction. Explicitly, the unperturbed spin-up and spin-down wave functions have the standard form

$$
\begin{aligned}
& \chi_{\uparrow}=\frac{\sqrt{N}}{\sqrt{2 \pi L_{x}}} \mathrm{e}^{\mathrm{i} k_{x} x}\left(\begin{array}{l}
1 \\
0
\end{array}\right) \chi(z), \\
& \chi_{\downarrow}=\frac{\sqrt{N}}{\sqrt{2 \pi L_{x}}} \mathrm{e}^{\mathrm{i} k_{x} x}\left(\begin{array}{l}
0 \\
1
\end{array}\right) \chi(z),
\end{aligned}
$$

where

$$
\chi(z)=\left(\mathrm{e}^{\mathrm{i} k_{z} z}+R \mathrm{e}^{-\mathrm{i} k_{z} z}\right)_{\mid z \leq 0}+\left(T \mathrm{e}^{\mathrm{i} q_{z} z}\right)_{\mid z>0} .
$$

Along the $x$ direction the functions are normalized to the length $L_{x}$, while along the $z$ direction we aim at the normalization to the delta function $\delta\left(k_{z}-k_{z}^{\prime}\right)$ and keep the normalization coefficient $\sqrt{N}$, where $N$ is to be determined.

Now we consider the boundary conditions at the interface. There is

and

$$
\left.\chi\right|_{+_{0}}=\left.\chi\right|_{-0},
$$

$$
\left(\frac{1}{m_{B}^{*}} \frac{\partial \chi}{\partial z}\right)_{+_{0}}=\left(\frac{1}{m_{A}^{*}} \frac{\partial \chi}{\partial z}\right)_{-_{0}} .
$$

This gives for both spin functions

$$
\frac{\mathrm{i} k_{z}(1-R)}{m_{A}^{*}}=\frac{\mathrm{i} T q_{z}}{m_{B}^{*}},
$$

and finally

$$
R=\frac{\left(k_{z}-q_{z} M\right)}{\left(k_{z}+q_{z} M\right)} .
$$

where $M=m_{A}^{*} / m_{B}^{*}$.

We assume that initially the electrons are characterized by the energy $E$ and the wave vector $k_{x}$. Since in the region $A$ there is $V(z)=0$, the energy of incoming electrons is $E=\hbar^{2}\left(k_{x}^{2}+k_{z}^{2}\right) / 2 m_{A}^{*}$, so we have

$$
k_{z}^{2}=\left(E \frac{2 m_{A}^{*}}{\hbar^{2}}-k_{x}^{2}\right),
$$

while in the barrier region $B$ there is

$$
q_{z}^{2}=\left[\left(E-V_{b}\right) \frac{2 m_{B}^{*}}{\hbar^{2}}-k_{x}^{2}\right] .
$$

Now we calculate the normalization of $\chi(z)$.

$$
\begin{aligned}
& \frac{N}{2 \pi} \int_{0}^{\infty} \mathrm{d} k_{z}^{\prime} \int_{-\infty}^{0} \mathrm{~d} z\left(\mathrm{e}^{\mathrm{i} k_{z} z}+R \mathrm{e}^{-\mathrm{i} k_{z} z}\right) \\
& \quad \times\left(\mathrm{e}^{-\mathrm{i} k_{z}^{\prime} z}+R^{\prime *} \mathrm{e}^{\mathrm{i} k_{z}^{\prime} z}\right)
\end{aligned}
$$

$$
+\frac{N}{2 \pi} \int_{0}^{\infty} \mathrm{d} q_{z}^{\prime} \int_{0}^{\infty} \mathrm{d} z T \mathrm{e}^{\mathrm{i} q_{z}} T^{*} \mathrm{e}^{-\mathrm{i} q_{z}^{\prime} z}=1 .
$$

The final formula for $N$ depends on the relation between the electron energy $E$ and $V_{b}+\hbar^{2} k_{x}^{2} / 2 m_{B}^{*}$.

a) The first case is: $E<V_{b}+\hbar^{2} k_{x}^{2} / 2 m_{B}^{*}$, so that $q_{z}=\mathrm{i}\left|q_{z}\right|$ according to Eq. (18). The function in the barrier $T e^{-\left|q_{z}\right| z}$ decays fast and we can neglect the term proportional to $T$. Consequently, $\chi(z)$ is nonzero for $-\infty<z \leq 0$. The normalization condition is

$$
\begin{aligned}
& \frac{N}{2} \int_{0}^{\infty} \mathrm{d} k_{z}^{\prime}\left(\left(1+R R^{\prime *}\right) \delta\left(k_{z}-k_{z}^{\prime}\right)\right. \\
& \left.\quad-\left(R+R^{\prime *}\right) \delta\left(k_{z}+k_{z}^{\prime}\right)\right)=1 .
\end{aligned}
$$

The second term gives no contribution to the integral since both $k_{z}, k_{z}^{\prime}>0$. The first term imposes $k_{z}^{\prime}=k_{z}$, then from Eq. (16) there is $1+R R^{\prime *}=2$, so that finally $N=1$. It should be noted that the integration over $k_{z}^{\prime}$ is related to the correct dimension of energy in Eq. (3), as mentioned above.

b) The second case is: $E>V_{b}+\hbar^{2} k_{x}^{2} / 2 m_{B}^{*}$, so that $q_{z}, R, T$ are real. The function $\chi(z)$ is nonzero for $-\infty<$ $z<\infty$. The normalization condition is

$$
\begin{aligned}
& \frac{N}{2}\left[\int_{0}^{\infty} \mathrm{d} k_{z}^{\prime}\left(1+R R^{\prime}\right) \delta\left(k_{z}-k_{z}^{\prime}\right)\right. \\
& \left.\quad+\int_{0}^{\infty} \mathrm{d} q_{z}^{\prime} T T^{\prime} \delta\left(q_{z}-q_{z}^{\prime}\right)\right]=1 .
\end{aligned}
$$

The first term gives $1+R^{2}$ and the second one gives $T^{2}=$ $(1+R)^{2}$. Finally there is $N=\left(k_{z}+M q_{z}\right)^{2} /\left(3 k_{z}^{2}+M^{2} q_{z}^{2}\right)$.

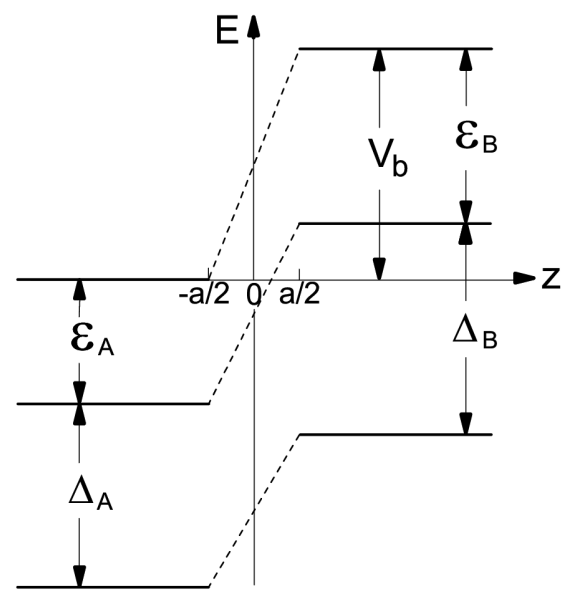

Fig. 1. One-dimensional potential barrier used for the calculation of spin-orbit energy (schematically). Energy gaps and spin-orbit energies in regions $\mathrm{A}$ and $\mathrm{B}$ are indicated.

To calculate the spin splitting determined by the average value of $\left|\widehat{K}_{\text {SIA }}^{\uparrow \downarrow}\right|=\Delta$, we take into account the valence offsets contained in the $\tilde{\varepsilon}_{i}(z)$ and $\tilde{f}_{i}(z)$ functions. We will assume here that the potential barrier is a steep but linear function of $z$, extending from $-a / 2$ to $a / 2$, see Fig. 1 . After some manipulation the expression for $\Delta$ is brought to the form 


$$
\begin{aligned}
\Delta & =\int_{0}^{\infty} \mathrm{d} k_{z}^{\prime} \int_{-a / 2}^{a / 2} \mathrm{~d} z\left|\chi_{\downarrow}^{*}\left(z, k_{z}^{\prime}\right) \widehat{K}_{\mathrm{SIA}} \chi_{\uparrow}\left(z, k_{z}\right)\right|= \\
& \frac{N k_{x}}{2} \frac{\left(\eta_{B}-\eta_{A}\right)}{a} \int_{0}^{\infty} \mathrm{d} k_{z}^{\prime}\left[1+R^{*}\left(k_{z}^{\prime}\right)\right]\left[1+R\left(k_{z}\right)\right] \\
& \times \frac{2}{2 \pi} \frac{\sin \left[\left(k_{z}-k_{z}^{\prime}\right) a / 2\right]}{\left(k_{z}-k_{z}^{\prime}\right)}= \\
& \frac{N k_{x}}{2} \frac{\left(\eta_{B}-\eta_{A}\right)}{a} \int_{0}^{\infty}\left[1+R^{*}\left(K_{z}^{\prime}\right)\right]\left[1+R\left(K_{z}\right)\right] \\
& \times \frac{\sin \left[\left(K_{z}-K_{z}^{\prime}\right) D\right]}{\pi\left(K_{z}-K_{z}^{\prime}\right)} \mathrm{d} K_{z}^{\prime} .
\end{aligned}
$$

By changing the variable $k_{z}$ into $K_{z}=k_{z} a / 2 D, k_{z}^{\prime}$ into $K_{z}^{\prime}=k_{z}^{\prime} a / 2 D,\left|q_{z}\right| M$ into $\left|Q_{z}\right|=\left|q_{z}\right| M a / 2 D$ and $\left|q_{z}^{\prime}\right|$ into $\left|Q_{z}^{\prime}\right|=\left|q_{z}^{\prime}\right| M a / 2 D$, where $D$ is a large number and $R\left(K_{z}\right)=\left(K_{z}-Q_{z}\right) /\left(K_{z}+Q_{z}\right)$, the last factor in the integrand of Eq. (22) can be replaced to a very good approximation by $\delta\left(K_{z}-K_{z}^{\prime}\right)$. Then the integration over $\mathrm{d} K_{z}^{\prime}$ in Eq. (22) can be carried out and the final result for the case $E<V_{b}+\hbar^{2} k_{x}^{2} / 2 m_{B}^{*}$ is

$$
\Delta_{E<}=\frac{k_{x}\left(\eta_{B}-\eta_{A}\right)}{2 a} \frac{4 k_{z}^{2}}{\left(k_{z}^{2}+\left|q_{z}\right|^{2} M^{2}\right)},
$$

while for the case $E>V_{b}+\hbar^{2} k_{x}^{2} / 2 m_{B}^{*}$ there is

$$
\Delta_{E>}=\frac{k_{x}\left(\eta_{B}-\eta_{A}\right)}{2 a} \frac{4 k_{z}^{2}}{\left(3 k_{z}^{2}+q_{z}^{2} M^{2}\right)} .
$$

The spin splitting is $2 \Delta$, see Eq. (8) and Fig. 2.

It can be seen that the spin splitting of the conduction band due to the SIA mechanism is proportional to the spin-orbit energies in the valence bands contained in $\eta_{B}-\eta_{A}$ term. Also, $\Delta$ depends on $k_{z}$ and $q_{z}$, which characterize the incoming electrons. In the published literature one deals with the Bychkov-Rashba spin splitting in quantum wells, where $\Delta$ is related to the electron wave function in the well. The unusual feature of our situation is that we do not deal with the well but with a barrier, so the wave function is replaced by the incoming and returning plane waves and the penetrating component.

\section{Electron reflection and penetration}

As to the wave functions perturbed by the SOI, it can be seen from Eqs. (3) and (6) that, for $k_{y}=0$, the two components of the $\widehat{K}_{\text {SIA }}$ operator are of the form $\widehat{K}_{\text {SIA }}=k_{x} f(z) \widehat{\sigma}_{y}$. This corresponds exactly to the general form given in Eq. (1). Thus the matrix element is $K_{\text {SIA }}^{\uparrow \downarrow} \sim \sigma_{y}^{\uparrow \downarrow}=-\mathrm{i}$ so that, according to the two-state perturbation procedure, in which the two perturbed functions are given by a sum and a difference of unperturbed functions, the perturbed spin functions are $(1 / \sqrt{2})(1, i)^{\mathrm{T}}$ and $(1 / \sqrt{2})(1,-i)^{\mathrm{T}}$, i.e. they are the eigenfunctions of $\widehat{\sigma}_{y}$ operator for the spin-up and spin-down states quantized along the $y$ direction. In the following we refer to these states as the "spin-up" and "spin-down" states, respectively.

Since the components of $\widehat{K}_{\text {SIA }}$ in Eq. (3) are proportional to $\widehat{\sigma}_{y}$, it is clear that the complete Hamiltonian commutes with $\widehat{\sigma}_{y}$. This means that the projection of electron spin on the $y$ direction is a constant of the motion. Since, on the other hand, in our case the perturbed wave functions are the eigenfunctions of $\widehat{\sigma}_{y}$, one concludes that the electron spin (on the $y$ direction) is a constant of the motion, so that spin-flip reflections from the barrier cannot occur. This conclusion has been confirmed by explicit calculations of the reflected amplitudes which, in case of spin-flip, vanish.

Let $\Psi_{1}$ describe a spin-up electron with the incoming wave vector $k_{z}^{\prime}>0$. The returning electron, reflected without the change of spin, has the wave vector $-k_{z}^{\prime}$ and the penetrating component is characterized by $q_{z}^{\prime}$. The spin-up electron energy is

$$
E_{1}=\frac{\hbar^{2} k^{2}}{2 m_{A}^{*}}+\Delta,
$$

where $k^{\prime 2}=k_{x}^{2}+k_{z}^{\prime 2}$. For the spin-down electron there is

$$
E_{2}=\frac{\hbar^{2} k^{\prime \prime 2}}{2 m_{A}^{*}}-\Delta .
$$

where $k^{\prime \prime 2}=k_{x}^{2}+k_{z}^{\prime \prime 2}$. The corresponding spin-up and spin-down wave functions are

$$
\begin{aligned}
& \Psi_{1}=\frac{\mathrm{e}^{\mathrm{i} k_{x} x}}{\sqrt{4 \pi L_{x}}}\left(\begin{array}{c}
1 \\
\mathrm{i}
\end{array}\right) \\
& \times\left\{\left[\mathrm{e}^{\mathrm{i} k_{z}^{\prime} z}+R \mathrm{e}^{-\mathrm{i} k_{z}^{\prime} z}\right]_{\mid z \leq 0}+\left[T \mathrm{e}^{\mathrm{i} q_{z}^{\prime} z}\right]_{\mid z>0}\right\}, \\
& \Psi_{2}=\frac{\mathrm{e}^{\mathrm{i} k_{x} x}}{\sqrt{4 \pi L_{x}}}\left(\begin{array}{c}
1 \\
-\mathrm{i}
\end{array}\right) \\
& \times\left\{\left[\mathrm{e}^{\mathrm{i} k_{z}^{\prime \prime} z}+P \mathrm{e}^{-\mathrm{i} k_{z}^{\prime \prime} z}\right]_{\mid z \leq 0}+\left[F \mathrm{e}^{\mathrm{i} q_{z}^{\prime \prime} z}\right]_{\mid z>0}\right\},
\end{aligned}
$$

where the second terms correspond to the returning and the third to the penetrating components. Let us assume that in an experiment the spin-up and spin-down electrons have the same total energy $E=E_{1}=E_{2}$. Since $k_{x}$ does not change, one gets from Eq. (25) for the spin-up electron

$$
\begin{aligned}
& {k_{z}^{\prime}}^{2}=(E-\Delta) \frac{2 m_{A}^{*}}{\hbar^{2}}-k_{x}^{2}, \\
& {q_{z}^{\prime 2}}^{2}=\left(E-\Delta-V_{b}\right) \frac{2 m_{B}^{*}}{\hbar^{2}}-k_{x}^{2},
\end{aligned}
$$

and from Eq. (26) for the spin-down electron

$$
\begin{aligned}
& k_{z}^{\prime \prime 2}=(E+\Delta) \frac{2 m_{A}^{*}}{\hbar^{2}}-k_{x}^{2}, \\
& q_{z}^{\prime \prime 2}=\left(E+\Delta-V_{b}\right) \frac{2 m_{B}^{*}}{\hbar^{2}}-k_{x}^{2} .
\end{aligned}
$$

There follow from Eqs. (30) and (32) unexpected and interesting possibilities. One usually expects that, when the incoming electron has the energy $E$ larger than the barrier height $V_{b}$, it can move above the barrier with a nonvanishing amplitude. However, it follows from Eq. (30) that a spin-up electron can have $E>V_{b}$ but still, due to the appearance of $\Delta$ with the minus sign, 
the resulting $q_{z}^{\prime 2}$ is negative, so that $q_{z}^{\prime}$ is imaginary. According to Eq. (27) this means that the penetrating wave is quickly damped, while the spin-up wave is almost totally reflected. On the other hand, one usually expects that, if $E<V_{b}$, the electron is almost completely reflected, while the penetrating wave is quickly damped. However, it follows from Eq. (32) that a spin-down electron can have $E<V_{b}$ and still, due to the appearance of $\Delta$ with the plus sign, the resulting $q_{z}^{\prime \prime 2}$ is positive, so that $q_{z}^{\prime \prime}$ is real. According to Eq. (28) this means that the penetrating wave propagates in the barrier with the smaller amplitude but without damping! Suppose now that incoming electrons have the energy $E>V_{b}$ and are not spin polarized. One can then express their spins as combinations of the spin-up and spin-down components. It is then possible that the spin-up components are damped in the barrier and almost totally reflected from the barrier, while the spin-down components propagate without damping. It is clear that this situation can serve as a spin-polarizing device. Alternatively, for $E<V_{b}$ the spin-up component may be almost totally reflected, while the spin-down components can propagate in the barrier and are only partly reflected. This again can serve as a spin-polarizing device. In the next section we give numerical estimations of such situations for chosen examples.

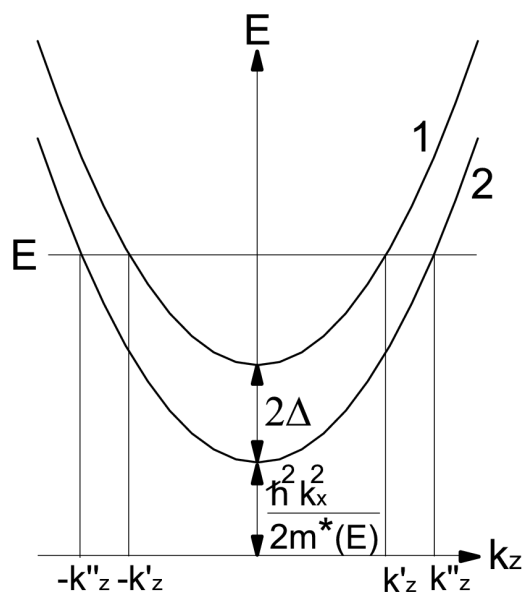

Fig. 2. Electron energy versus wave vector $k_{z}$ for spinup and spin-down states (schematically). The spinorbit energy is $\Delta$. For a spin-conserving elastic reflection of spin-up electron the outgoing $k_{z}^{\prime}$ is equal to $-k_{z}^{\prime}$. Situation for an initial spin-down electron with the initial wave vector $k_{z}^{\prime \prime}$ is also shown.

\section{Estimations}

We estimate numerical values of amplitudes by considering the specific barrier: $\mathrm{InSb} / \mathrm{In}_{0.91} \mathrm{Al}_{0.09} \mathrm{Sb}$. In Table I we quote the band parameters of the involved narrow-gap semiconductor materials plus the offset $V_{b}$ of the heterojunction. All the other parameters can be inferred from the given values. In Table II we give the effective masses calculated for the electron energies $E$ in the regions $A$ and $B$. It should be reminded that the wave vectors $q_{z}^{\prime}$ and $q_{z}^{\prime \prime}$ refer to the spin-up and spin-down electrons, respectively.

TABLE I

Material parameters used for $\mathrm{InSb} / \mathrm{In}_{0.91} \mathrm{Al}_{0.09} \mathrm{Sb}$ barrier. The lattice constant $a$ is taken as an average value between $\mathrm{InSb}$ and $\mathrm{In}_{0.91} \mathrm{Al}_{0.09} \mathrm{Sb}$.

\begin{tabular}{c|c|c}
\hline \hline & $\mathrm{InSb}$ & $\mathrm{In}_{0.91} \mathrm{Al}_{0.09} \mathrm{Sb}$ \\
\hline$E_{\mathrm{g}}[\mathrm{eV}]$ & -0.2466 & -0.426 \\
$\Delta_{0}[\mathrm{eV}]$ & -0.8419 & -0.777 \\
$m_{0}^{*} / m_{0}$ & 0.0143 & 0.0234 \\
$C$ & -1.496 & -1.3685 \\
$E_{\mathrm{p}}[\mathrm{eV}]$ & 23.4 & 23.4 \\
$a\left(10^{8} \mathrm{~cm}\right)$ & 6.4 & 6.4 \\
$V_{b}[\mathrm{eV}]$ & - & 0.111
\end{tabular}

TABLE II

Heterojunction parameters used for $\mathrm{InSb} / \mathrm{In}_{0.91} \mathrm{Al}_{0.09} \mathrm{Sb}$ barrier. The effective masses $m_{A}^{*} / m_{0}$ and $m_{B}^{*} / m_{0}$ are calculated for given electron energies $E$.

\begin{tabular}{c|c|c}
\hline \hline & $\mathrm{InSb}$ & $\mathrm{In}_{0.91} \mathrm{Al}_{0.09} \mathrm{Sb}$ \\
\hline$E[\mathrm{eV}]$ & 0.08 & 0.08 \\
$m_{A}^{*} / m_{0}$ & 0.01854 & - \\
$m_{B}^{*} / m_{0}$ & - & 0.02184 \\
\hline $\mathrm{E}[\mathrm{eV}]$ & 0.13 & 0.13 \\
$m_{A}^{*} / m_{0}$ & 0.02113 & - \\
$m_{B}^{*} / m_{0}$ & - & 0.02435
\end{tabular}

In Tables III and IV we quote characteristics of reflected and penetrating waves, as calculated for different initial sets of parameters. The initial sets comprise electron energy $E$ for $E<V_{b}+\hbar^{2} k_{x}^{2} / 2 m_{B}^{*}$ (Table III) and $E>V_{b}+\hbar^{2} k_{x}^{2} / 2 m_{B}^{*}$ (Table IV) and the wave vector $k_{x}$ (two values). In entries for $k_{x}=0.35 \times 10^{6} \mathrm{~cm}^{-1}$ we deal with the "standard" case in which $k_{z}^{\prime}$ and $k_{z}^{\prime \prime}$ have real values, while $q_{z}^{\prime}$ and $q_{z}^{\prime \prime}$ have imaginary values for $E<$ $V_{b}+\hbar^{2} k_{x}^{2} / 2 m_{B}^{*}$ and real values for $E>V_{b}+\hbar^{2} k_{x}^{2} / 2 m_{B}^{*}$. An interesting and unexpected situation is described in the entries for $k_{x}=1 \times 10^{6} \mathrm{~cm}^{-1}$. It follows from Tables I and III that, for the incoming energy $E=80 \mathrm{meV}$ of the spin-down electron and $V_{b}=111 \mathrm{meV}$, there is $E<V_{b}+\hbar^{2} k_{x}^{2} / 2 m_{B}^{*}$, i.e. the kinetic electron energy is smaller than the barrier height. According to the intuitive expectations, it should be a "normal" case in which the wave penetrating the barrier quickly decays with the distance $z$. However, the calculated spin-orbit energy for this case is $\Delta=58 \mathrm{meV}$, so that $E+\Delta=138 \mathrm{meV}$ is higher than $V_{b}$. As a consequence, it follows from Eq. (32) that the resulting $q_{z}^{\prime \prime}=0.736 \times 10^{6} \mathrm{~cm}^{-1}$ is real. This means that the penetrating spin-down wave $\exp \left(\mathrm{i} q_{z}^{\prime \prime} z\right)$ propagates in the barrier without attenuation!

An alternative situation is created by an incoming spin-up electron with the energy $E=130 \mathrm{meV}$, see Table IV. For the barrier $V_{b}=111 \mathrm{meV}$ there is $E>$ $V_{b}+\hbar^{2} k_{x}^{2} / 2 m_{B}^{*}$, so that the incoming electron moves in 
TABLE III

Case $E<V_{b}+\hbar^{2} k_{x}^{2} / 2 m_{B}^{*}$. Spin-orbit energies and reflection characteristics for spin-up and spin-down electrons, as calculated for $E=0.08 \mathrm{eV}$ and $V_{b}=0.111 \mathrm{eV}$, and given values of $k_{x}$.

\begin{tabular}{c|c|c}
\hline \hline$k_{x}\left[10^{-6} / \mathrm{cm}\right]$ & 0.35 & 1.0 \\
$\Delta[\mathrm{eV}]$ & 0.025 & 0.058 \\
\hline$q_{z}^{\prime}\left[10^{-6} / \mathrm{cm}\right]$ & $\mathrm{i} 1.828$ & $\mathrm{i} 2.47$ \\
$R$ & $0.03-\mathrm{i} 0.9995$ & $-0.968-\mathrm{i} 0.25$ \\
$R C_{1}$ & 1 & 1 \\
$T C_{1}$ & 0 & 0 \\
\hline$q_{z}^{\prime \prime}\left[10^{-6} / \mathrm{cm}\right]$ & $\mathrm{i} 0.676$ & 0.736 \\
$P$ & $0.89-\mathrm{i} 0.455$ & 0.585 \\
$R C_{2}$ & 1 & 0.342 \\
$T C_{2}$ & 0 & 0.658
\end{tabular}

TABLE IV

Case $E>V_{b}+\hbar^{2} k_{x}^{2} / 2 m_{B}^{*}$. The same as in Table III but for $E=0.13 \mathrm{eV}$ and $V_{b}=0.111 \mathrm{eV}$.

\begin{tabular}{c|c|c}
\hline \hline$k_{x}\left[10^{-6} / \mathrm{cm}\right]$ & 0.35 & 1.0 \\
$\Delta[\mathrm{eV}]$ & 0.0085 & 0.025 \\
\hline$q_{z}^{\prime}\left[10^{-6} / \mathrm{cm}\right]$ & 0.74 & 11.176 \\
$R$ & -0.600 & $0.645-\mathrm{i} 0.764$ \\
$R C_{1}$ & 0.36 & 1 \\
$T C_{1}$ & 0.64 & 0 \\
\hline$q_{z}^{\prime \prime}\left[10^{-6} / \mathrm{cm}\right]$ & 1.279 & 1.346 \\
$P$ & 0.425 & 0.405 \\
$R C_{2}$ & 0.181 & 0.164 \\
$T C_{2}$ & 0.819 & 0.836
\end{tabular}

principle above the barrier. However, for this case there is $\Delta=25 \mathrm{meV}$, so that $E-\Delta<V_{b}+\hbar^{2} k_{x}^{2} / 2 m_{B}^{*}$ and, according to Eq. (30), the resulting $q_{z}^{\prime}=\mathrm{i} 1.176 \times 10^{6} / \mathrm{cm}$ is imaginary. This means that the continuing spin-up component quickly decays above the barrier and there is a total reflection of the spin-up wave from the barrier.

It is instructive to calculate not only the amplitudes, but also reflection and transmission coefficients of various waves. A transmission coefficient $\mathrm{TC}$ is defined as $T C=$ $\left|J_{\text {tr }} / J_{\text {in }}\right|$ and the reflection coefficient as $R C=\left|J_{\mathrm{re}} / J_{\text {in }}\right|$, where $J_{\text {in }}$ is the incoming current. According to Eqs. (27) and (28), we have for the incoming spin-up electron: $T C_{1}=|T|^{2} q_{z}^{\prime} m_{A}^{*} / k_{z}^{\prime} m_{B}^{*}$ and $R C_{1}=|R|^{2}$ while for the incoming spin-down electron: $T C_{2}=|F|^{2} q_{z}^{\prime \prime} m_{A}^{*} / k_{z}^{\prime} m_{B}^{*}$ and $R C_{2}=|P|^{2}$. For imaginary $q_{z}^{\prime}$ or $q_{z}^{\prime \prime}$ we have the decaying waves in the barrier, i.e. $J_{\mathrm{tr}}=0$ and $T C=0$. In the last entries of Tables III and IV we give the values of $R C$ and $T C$, as calculated from the above relations. It can be seen that $R C+T C=1$, as it should be. One can also see that in the interesting two cases: the nonattenuated transmitted wave for $E<V_{b}+\hbar^{2} k_{x}^{2} / 2 m_{B}^{*}$ (Table III) has a large transmission coefficient $T C_{2}=0.658$, while for electron with $E>V_{b}+\hbar^{2} k_{x}^{2} / 2 m_{B}^{*}$ (Table IV) the transmitted wave is attenuated in the barrier and has the transmission coefficient $T C_{1}=0$.

As mentioned above, the considered system can serve as a source of spin-polarized electron beams since it spatially separates electrons with specifically oriented spins. A state with unpolarized electron spin can be represented as a combination of the spin-up and spindown components. If, in the first considered process (for $k_{x}=1.0 \times 10^{6} \mathrm{~cm}^{-1}$ in Table III), the spin-up component is totally reflected, while the spin-down component is partly transmitted without attenuation, it is clear that the transmitted electrons will have the spin-down polarization. Similarly, in the second considered process (for $k_{x}=1.0 \times 10^{6} \mathrm{~cm}^{-1}$ in Table IV), the spin-up component is totally reflected, while the spin-down component is partially transmitted without attenuation, so that the transmitted electrons will have again the spin-down polarization.

\section{Discussion}

We first examine the approximations used in our approach. The description of the potential barrier is somewhat ambiguous since we sometimes use a vertical barrier and, when calculating the spin-orbit energy for unbound electrons, a barrier of finite slope. As we mentioned in Sect. 2, the latter assumption was necessary in order to avoid an infinite electric field. However, this ambiguity is not troublesome since one needs a steep barrier to obtain sizable spin splittings. On the other hand, a narrow barrier (on the order of interatomic distance) is in a certain contradiction with the validity of the $\boldsymbol{k} \cdot \boldsymbol{p}$ theory. The latter requires that the potential changes be slow compared to the lattice period. Fortunately, it is known that the $\boldsymbol{k} \cdot \boldsymbol{p}$ theory usually gives better results than it is supposed to. Also, when calculating SOE we assume in Eq. (22) that the reflection amplitude $R$ does not depend on $z$. All in all, it is possible that our calculations of SOE for unbound electrons are not very precise. This, however, should not influence qualitatively our results. To the best of our knowledge, a calculation of the BychkovRashba spin splitting for unbound electrons has not been attempted before.

As it was mentioned in Sect. 2, the spin-flip ballistic reflection of electrons from a potential barrier was experimentally realized by Chen and coworkers in $\mathrm{InSb} / \mathrm{InAlSb}$ and InAs/AlGaSb heterostructures [10, 12, 14]. In their experiments, the authors used a "triangular geometry": two sides of a triangle with slits were employed to inject and detect electron beams, the third side was utilized as a barrier. An external magnetic field transverse to the 2D plane of the heterostructure was used to direct the reflected beams to the detecting slit. A theoretical description of these experiments was presented mostly in Ref. [11]. The authors assumed that the spin splitting of electron energies due to the SOI had two origins: the structural asymmetry of the quantum well and the barrier. However, it was then incorrectly concluded that the Bychkov-Rashba spin splitting of electron energies due to the asymmetric well was proportional to the electric field in the conduction band and that the spin splittings caused by the two origins were governed by the same material parameter. The calculation of spin splitting due to 
the SO interaction of unbound electrons with the barrier was not attempted. Teodorescu and Winkler [15] in their analysis of spin-dependent reflections from an impenetrable barrier used as an origin of the Bychkov-Rashba spin splitting only the inversion asymmetry of the well. In the above arrangements the spin-flip reflections were possible because they had the reflecting barrier in addition along the $x$ direction, while the spin resulting from the asymmetry of the quantum well (grown parallel to the $z$ axis) was directed along the $y$ axis.

In the work on the spin separation in $2 \mathrm{D}$ cyclotron motion (Ref. [16]) and transverse electron focusing in 2D systems with the SOI (Ref. [17]), the Bychkov-Rashba splitting for unbound electrons was taken into account only phenomenologically. In this connection we mention that the SOI related to the barrier can separate spins in the presence of an external magnetic field $\boldsymbol{B}$ transverse to the electron motion ( $x-y$ plane in our geometry). Suppose that all incoming electrons have the Fermi energy $E_{\mathrm{F}}$. Then the spin-up and spin-down components have the kinetic energy $m^{*} v_{ \pm}^{2}=E_{\mathrm{F}} \mp \Delta$, respectively. The resulting Lorentz force in the two cases is different: $\boldsymbol{F}_{ \pm}=e\left(\boldsymbol{v}_{ \pm} \times \boldsymbol{B}\right)$, so that the corresponding electron trajectories, including reflections from the barrier, will be different.

It should be emphasized that, as follows from our Eq. (23) and Ref. [2], the Bychkov-Rashba splitting for unbound and bound electrons bear some similarities but are also marked by important differences. In both cases it is necessary to have the SOI and the structure inversion asymmetry. In both cases it is necessary to have a transverse motion and the resulting spin splitting is proportional to the transverse momentum. On the other hand, for the unbound electrons the spin splitting depends on the longitudinal momentum $\hbar k_{z}$, while for the bound electrons this momentum is "frozen" in the electron wave function of the quantum well and it does not explicitly appear. Also, as follows from Eqs. (23) and (24), for the unbound electrons even the functional forms of $\Delta$ are different for $E<V_{b}+\hbar^{2} k_{x}^{2} / 2 m_{B}^{*}$ and $E>V_{b}+\hbar^{2} k_{x}^{2} / 2 m_{B}^{*}$ situations.

\section{Summary}

We described spin-conserving opaque reflections of $2 \mathrm{D}$ electrons from a barrier in $\mathrm{InSb} / \mathrm{InAlSb}$ heterostructure. In such a system the electric field of the barrier is the only source of spin splitting in the presence of the spinorbit interaction. Formulae for the Bychkov-Rashba spin splitting are calculated for unbound electrons. Amplitudes are calculated for reflected and penetrating electron beams. It is shown that, in the considered simple geometry, the spin-flip electron reflections are not possible. Two unexpected possibilities are predicted due to the spin-orbit interaction: (a) non-attenuated electron propagation in the barrier when the latter is higher than the kinetic energy of incoming electrons, (b) total reflection of electrons whose energies exceed barrier's height. It is pointed out that the considered system can serve as a source or filter of spin-polarized electron beams.

\section{Acknowledgments}

We are grateful to Dr. T.M. Rusin for elucidating discussions.

\section{References}

[1] G. Dresselhaus, Phys. Rev. 100, 580 (1955).

[2] W. Zawadzki, P. Pfeffer, Semicond. Sci. Technol. 19, R1 (2004).

[3] Y.A. Bychkov, E.I. Rashba, J. Phys. C 17, 6039 (1984).

[4] L. Wissinger, U. Rossler, R. Winkler, B. Jusserand, D. Richards, Phys. Rev. B 58, 15375 (1998).

[5] P. Pfeffer, Phys. Rev. B 59, 15902(1999).

[6] R. Winkler, Spin-Orbit Coupling Effects in TwoDimensional Electron and Hole Systems, Springer Tracts in Modern Physics, Vol. 191, Springer, Berlin 2003.

[7] W. Zawadzki, P. Pfeffer, Phys. Rev. B 64, 235313 (2001).

[8] V.M. Ramaglia, D. Bercioux, V. Cataudella, G. De. Filippis, C.A. Perroni, J. Phys., Condens. Matter 16 , 9143 (2004)

[9] M. Khodas, M. Shekhter, A.M. Finkelstein, Phys. Rev. Lett. 92, 086602 (1999).

[10] H. Chen, J.J. Heremans, J.A. Peters, J.P. Dulka, A.O. Govorov, N. Goel, S.J. Chung, M.B. Santos, arXiv:0308569, (2003)

[11] A.O. Govorov, A.V. Kalameitsev, J.P. Dulka, Phys. Rev. B 70, 245310 (2004).

[12] H. Chen, J.J. Heremans, J.A. Peters, A.O. Govorov, N. Goel, S.J. Chung, M.B. Santos, Appl. Phys. Lett. 86, 032113 (2005)

[13] G. Usaj, C.A. Balseiro, Europhys. Lett. 72, 631 (2005).

[14] H. Chen, J.A. Peters, Y. Pan, J.J. Heremans, N. Goel, S.J. Chung, M.B. Santos, W. Van Roy, G. Borghs, Physica E 34, 374 (2006).

[15] V. Teodorescu, R. Winkler, Phys. Rev. B 80 041311R (2009).

[16] L.R. Rokhinson, V. Larkina, Y.B. Lyanda-Geller, L.N. Pfeiffer, K.W. West, Phys. Rev. Lett. 93 146601 (2004).

[17] G. Usaj, C.A. Balseiro, Phys. Rev. B 70, 041301R (2004). 